\title{
Bubble Dynamics on a Heated Surface
}

\author{
M. Kassemi* and N. Rashidnia** \\ NASA Lewis Research Center \\ Cleveland, Ohio
}

$\exists t^{\prime}$

\begin{abstract}
In this work, we study the combined thermocapillary and natural convective flow generated by a bubble on a heated solid surface. The interaction between gas and vapor bubbles with the surrounding fluid is of interest for both space and ground-based processing. On earth, the volumetric forces are dominant, especially, in apparatuses with large volume to surface ratio. But in the reduced gravity environment of orbiting spacecraft, surface forces become more important and the effects of Marangoni convection are easily unmasked. In order to delineate the roles of the various interacting phenomena, a combined numerical-experimental approach is adopted.The temperature field is visualized using Mach-Zehnder interferometry and the flow field is observed by a laser sheet flow visualization technique. A finite element numerical model is developed which solves the two-dimensional momentum and energy equations and includes the effects of bubble surface deformation. Steady state temperature and velocity fields predicted by the finite element model are in excellent qualitative agreement with the experimental results. A parametric study of the interaction between Marangoni and natural convective flows including conditions pertinent to microgravity space experiments is presented. Numerical simulations clearly indicate that there is a considerable difference between $1-\mathrm{g}$ and low- $\mathrm{g}$ temperature and flow fields induced by the bubble.
\end{abstract}

\section{Introduction}

In many industrial situations which involve materials processing, manufacturing, boiling, and storage and management of liquids, the interaction between gas and vapor bubbles with the surrounding fluid is of great interest. This interaction is manifested by a fluid motion brought about by two co-existing and sometimes competing mechanisms; natural convection induced by the volumetric buoyancy force and Marangoni convection driven by the interfacial stresses along the bubble surface. Unlike natural convection which is driven by density differences generated by either temperature or concentration gradients in the bulk of the fluid, Marangoni convection is driven by surface tension forces brought about by temperature or concentration gradients (thermocapillary and solutocapillary convection, respectively) al ong the free surfaces of the fluid volume. On earth, the volumetric forces are dominant, especially, in apparatuses with large volume to surface ratio. But in the reduced gravity environment of orbiting space craft, surface forces become more important and the effects of Marangoni convection are easily unmasked.

In this work, we study the interaction between thermocapillary convection and natural convection generated by a bubble on a heated solid surface in both 1-g and low-g environments. In addition to its inherent scientific significance, this phenomenon is also of prime practical importance to materials processing and boiling processes.A Comprehensive review paper by Ostrach ${ }^{1}$ drew attention to the importance of Marangoni convection in materials processing and fluid management in space. As a result, there has been a significant volume of published work investigating the role of thermocapillary flows in the past decade. A category which has received much less attention includes studies of the interaction of bubbles attached to heated or cooled surfaces with the surrounding fluid. Need for low-gravity research in this area was demonstrated by a series of low-g and 1-g solidification experiments by Papazian and Wilcox ${ }^{2}$. They demonstrated that while during ground-based tests bubbles were seen to detach from the interface easily and float to the top of the melt, in low-gravity tests no detachment from the interface occurred and large voids were grown in the crystal. Larkin ${ }^{3}$ conducted the first numerical study of the thermocapillary effect around a hemispherical bubble placed on a solid wall subject to a constant heat flux. He obtained time-dependent numerical solutions for Marangoni numbers between 0 and 100,000 and Prandtl numbers between 1 and 5 . The results indicate that the flow builds up quickly and then gradually declines with time. Unfortunately, Larkin was unable to continue the solution until steady-state was reached because of the enormous computer time required, nor was he able to predict an oscillatory flow. It should be mentioned that Larkin did not include the effect of natural convection in his numerical model. Kao and Kenning ${ }^{4}$ extended the work of Larkin by taking into account heat transfer at the interface between a gas bubble and the surrounding liquid and studied the effect of surfactants on the flows. They covered a range of Marangoni numbers from 50 to 250,000 and Biot numbers from 0 to 5000 . Although the effects of natural convection were included in their analysis, they still did not predict flow and temperature oscillations. The aforemen-

* OAI/NASA Senior Research Scientist

** NYMA/NASA Senior Research Scientist 
tioned numerical results contradict recent experimental observations made by Raake et al. ${ }^{5}$ and Chun et al. ${ }^{6}$ and Rashidnia ${ }^{7}$ who, using interferometry, visualized thermal oscillations in the fluid surrounding a bubble at Marangoni numbers above 12,000 . The published numerical predictions are also not in accordance with the unsteady thermocapillary flow behavior reported at high Marangoni numbers in liquid bridges or free surface enclosures as described above.

\section{Mathematical Formulation}

Consider an enclosure containing a liquid (silicone oil) with a bubble placed on the inside of the top wall as shown in Fig. 1. The side walls are insulated and the temperature of the top and bottom walls are uniformly maintained at $T_{h}$ and $T_{c}$, respectively. Therefore, a thermally stratified state is established in the enclosure before the bubble is introduced. Once the bubble is positioned and the interface between the air and test liquid is formed, surface tension forces created by the temperature gradient along the interface will drive a thermocapillary convective flow. This thermocapillary flow disrupts the thermal stratification near the bubble resulting in significant temperature gradients and convection near the bubble surface.

A two dimensional model is developed to describe the fluid flow and heat transfer induced by the bubble. In this work, the Boussinesq approximation is used which is valid for small temperature differences $(\Delta T=4)$. All the material properties, except for the surface tension coefficient are assumed to be constant. The dependance of surface tension on temperature is experimentally determined and incorporated into the model. Following these assumptions, the flow in the enclosure is described by the continuity and momentum equations which are written in dimensionless form as

$$
\begin{gathered}
\nabla \cdot \vec{\nabla}=0 \\
\operatorname{Re}\left(\frac{\partial}{\partial t} \vec{\nabla}+\vec{\nabla} \cdot \nabla \vec{\nabla}\right)=-\nabla P+\nabla^{2} V-\left(\frac{G r}{R e}\right) \theta \hat{e}_{8}
\end{gathered}
$$

These equations are subject to nonslip condition at the top, bottom, and left boundaries, and symmetry conditions along the central axis of the enclosure.

The normal and tangential stress balances along the surface of the bubble are written as.

$$
\begin{gathered}
\sigma_{n}=2 \gamma H-P_{a} \\
\sigma_{t}=\nabla_{t} \theta
\end{gathered}
$$

Note that there are two driving forces for the flow. The first is due to the surface tension force which drives a vigorous tangential velocity along the bubble surface. The second is the buoyancy force which is driven by the density differences in the bulk of the fluid. Because both surface tension and density are functions of temperature, both of these driving forces couple the momentum equation to the energy equation.

The energy equation is written in dimensionless form as

$$
\operatorname{Re}\left(\frac{\partial \theta}{\partial t}+\nabla \cdot \nabla \theta\right)=\left(\frac{1}{P r}\right) \nabla^{2} \theta
$$

This equation is subject to insulated boundary conditions at the wall, symmetry condition at the center and prescribed temperatures at the top and bottom walls respectively:

$$
\begin{aligned}
& \theta=\theta_{h} \\
& \theta=\theta_{c}
\end{aligned}
$$

There are four important dimensionless parameters which govern the fluid flow and heat transfer in the enclosure. These are the Re, Gr, Pr, and Ca numbers. The first three dimensionless parameters can be combined using the definitions of Marangoni and Raleigh numbers as $M a=R e \cdot P r$ and $R a=G r \cdot P r$. The ratio of $\mathrm{Ra}$ and Ma numbers (most commonly referred to as the Bond number) is a measure of the relative importance of heat transfer by natural convection to heat transfer by thermocapillary convection.

To solve the problem numerically, a finite element model for the bubble is developed based on the simulation code 
Fidap. In this two-dimensional steady heat transfer and fluid flow model the bubble is treated as a flexible free surface with a specified contact angle with the top wall. The position of the interface is an unknown variable which is updated as the solution evolves. The boundary conditions on the interface are written as continuities of normal and tangential stresses and the kinematic constraint that requires the interface to always remain an interface. According to this scheme which is sometimes referred to as normal stress updating, the kinematic constraint and the balance of tangential stresses are used to satisfy the boundary conditions for the momentum equation and the normal stress balance is applied to determine the position of the interface. As a result, during a pseudo-transient solution, the position of all the nodes is adjusted according to the updated position of the interface. The equations are solved using a segregated approach in which each degree of freedom is tackled individually and the equations are, therefore, only loosely connected. This results in convergence rates which are lower than coupled solvers but the method has a large radius of convergence and is also not demanding on computer resources such as storage allocation which can be prohibitive due to the chustering of the nodes near the bubble (which is needed in order to resolve the very thin boundary layers formed at high Ma numbers).

\section{Experimental Setup and Procedures}

The experimental setup designed and constructed to quantify the thermocapillary flow generated by the bubble consists of three main components; the test cell and the injector, The Mach-Zehnder Interferometer (MZI), and the laser sheet flow visualization unit. The onset of the thermocapillary and natural convection flows is determined by observing the interferometric fringe patterns created by the temperature field and visualizing the velocity field near the bubble using a laser sheet. A schematic of the test cell which is made of large optical glass widows suitable for interferometric measurements is shown in Fig. 2. The enclosure has inside dimensions of $38 \mathrm{~mm} \times 19 \mathrm{~mm} \times 19 \mathrm{~mm}$ (w $x d x h)$. The transparent side walls are also suitable for laser light sheet illumination. The copper top and bottom walls are heated and cooled in order to establish the desired temperature gradients. Two reservoirs (not shown) are diagonally located in the comers of the upper copper wall to allow for expansion/contraction of the test liquid. These reservoirs are also used to supply and remove the fluid. There is a capillary opening with a connection to a syringe (not shown) at the center of the top plate for bubble injection. A depression is formed around the injection hole to hold various size bubbles. The chamber is filled with the test liquid. The fluid is heated from above and cooled from below by two independently controlled thermostatic circulation baths connected to the copper plates. When the desired temperature difference in the chamber is reached and steady-state conditions prevail, an air bubble of controlled size will be injected. Heat loses through the side walls and windows are negligible due to the vacuum in between the transparent double walls of the chamber (this can be confirmed from the flat interferometric fringe patterns shown in Fig 5). The entire diagnostic instrumentation and test cell, with the exception of the thermally controlled constant baths are installed on a vibration-isolation optical table for the ground-based experiments. The baths are connected to the test cell via flexible tygon tubing.

The flow fields are visualized with a low power $(5 \mathrm{~mW})$ HeNe laser light sheet at the meridian plane of the bubble. Neutrally buoyant particles, added to the test liquid, are used as flow tracers. The movement of the tracer particles are recorded by a time-lapse recorder on S-VHS. The temperature fields is visualized using MZI which has been successfully used in previous investigations with similar flow configurations ${ }^{6}$. This interferometric technique has been chosen because of its accuracy and sensitivity in pinpointing the different modes of oscillation. In this technique, light rays transversing through a phase object furnish an integral information about the refractive index distribution of the medium they have travelled through. For axisymmetric flow conditions the interferometric fringe patterns can be translated to temperature contours using Abel transformation?

\section{Results and Discussion}

\section{Numerical simulations}

In onder to gain insight into the flow and temperature fields generated by the bubble in a typical ground-based situation, numerical simulations were performed for silicone oil $(\mathrm{Pr}=8.4)$ as the test fluid. The steady-state results for the base case ( $R e=4300, G=2400)$ is presented in Fig 3. The streamlines in Fig. 3a indicate that three vortices exist in the enclosure. The strongest vortex driven by the thermocapillary force fills the top portion of the enclosure next to the bubble. It carries the fluid down along the surface of the bubble creating a strong boundary layer flow which is revealed by the packing of streamlines near the interface. The temperature contours around the bubble are distorted significantly by this vigorous flow as indicated in Fig 3b. At steady-state, the primary thermocapillary vortex co-exists with two additional vortices below the bubble. These secondary and tertiary vortices are driven by the buoyancy force brought about by unstable density gradients created as the hot fluid from the top of the enclosure flows into the colder region by thermocapillary convection. It is important to note that the secondary vortex rotates in the opposite direction of the primary vortex and impedes the enlargement of this vortex and the free flow of fluid into the lower regions of the enclosure. These numerical predictions are in close qualitative agreement with our flow visualization results 
shown in Fig. 6and the experimental flow and temperature fields reported by Raake et al. ${ }^{4}$. Finally, Fig. $3 \mathrm{c}$ indicates that the steady-state shape of the bubble (as determined by the balance of forces at the interface) is quite different from the initial hemispherical shape assumed at the beginning of the simulation. Again, there is an extremely good qualitative agreement between the flattened bubble shape predicted by the model and the experimental observations in our laboratory (Fig. 6)and by Raake et al. ${ }^{4}$ Our results indicate that with increasing Re number, the temperature along the bubble becomes more uniform, and consequently, the velocity of the fluid along the bubble surface which is driven by the temperature-dependant surface tension force is significantly rectuced. The largest $\mathrm{u}$ and $\mathrm{v}$ velocities at the interface occur for the Re=43 case which has the highest level of thermal stratification. Moreover, for the Re $=4300$ case, the flow attains its peak interfacial speed near the contact point between the bubble and the top wall. while for lower Re numbers, the peak speed shifts lower towards the central regions of the bubble.

Next, conditions pertinent to the microgravity environment are considered. Since, a low gravity environment is characterized by a reduced buoyancy force, decreased hydrostatic head, and negligible natural convection, the low-g shape of the bubble and the resulting thermocapillary flow and temperature fields will be also significantly different from the $1-\mathrm{g}$ cases discussed above. Again, we use numerical simulations to underscore this fact.

The steady-state flow and temperature fields generated by the bubble in a low-g environment $\left(g_{0}=10^{-4} \mathrm{~g}\right)$ are shown in Fig. 4. Just as in the 1-g situation, a vigorous thermocapillary flow is generated next to the bubble surface. Again, this strong flow will drastically modify the temperature profiles in the enclosure. But in contrast to the terrestrial examples presented in Fig. 3, this time, a natural convective flow will not ensue due to the reduced buoyancy force. As a result, the recirculating thermocapillary vortex will grow unopposed until it nearly fills the entire enclosure at steady-state. The streamlines of Fig. 4a clearly show that the microgravity flow pattern resembles a jet-like flow emanating from around the bubble and flowing downwards into the enclosure. As a result of this intense recirculating flow, the temperature field is greatly altered as depicted in Fig. 4b. The low-gravity bubble shape is plotted in Fig. 4c. Comparison with the bubble shape presented in Fig. 3c clearly shows the significant difference between low-g and 1$\mathrm{g}$ bubble shapes. In low gravity, the bubble is elongated rather than flattened (as in the $1-\mathrm{g}$ case) from its originally assumed hemispherical shape. Thus, in microgravity, the shape of the bubble, and the temperature and fluid flow fields are all drastically different from their terrestrial counterparts.

\section{Interferometric and Flow Visunlization Results}

The interferometric patterns established in the enclosure before the bubble is placed are shown in Fig. 5. When the bubble is introduced, both the flow and temperature fields are modified. At low Ma numbers the flow reaches steadystate conditions as discussed previously. An example of the steady-state interferometric patterns and flow field is included in Fig. 6 for $\mathrm{Ma}=3.6 \times 10^{4}$.

Our experimental results indicate that these steady state temperature and flow fields can be disrupted at higher Ma numbers. Initiation of periodic oscillations at larger Marangoni numbers is clearly indicated by again observing the basic thermocapillary flow and interferometric patterns. This periodic behavior is demonstrated by the sequence of interferometric fringe contours presented in Fig. 7 for $\mathrm{Ma}=9 \times 10^{4}$. The interferograms depict one full cycle of oscillation. Preliminary particle flow visualizations also show similar oscillations in the velocity distributions around the bubble. The flow and temperature fields for this case are axisymmetric. Our experience indicates that at still higher Marangoni numbers, the flow becomes nonperiodic and eventually will lead to chaotic patterns.

\section{Conclusions}

In this work a combined experimental numerical approach to investigate the fluid flow and temperature fields created by a bubble attached to a heated solid surface was presented. From the results obtained the following conclusions can be made.

1. Both experimental observation and numerical predictions indicate that the thermo-capillary flow induced by bubbles on earth is greatly influenced and affected by its inevitable interactions with buoyancy-driven convection.

2. steady-state numerical predictions indicate that the temperature and fluid fields generated by the bubble in lowgravity and its shape are significantly different from their ground-based counterparts. There has been no direct low-gravity experimental verification or confirmation of these numerical predictions.

3. Preliminary interferometric measurements in our laboratory demonstrate that the flow and temperature fields around the bubble can go through various oscillatory modes depending on the parametric space of the experiment. The nature and origin of these modes and their dependance on the interactions between natural and thermocapillary convection are as of yet not clearly known.

Results and conclusions obtained during ground-based experiments with regard to oscillatory convection cannot in any way be extrapolated to determine the behavior of the thermocapillary flow in space. Therefore extensive hand-inhand experiments and numerical modelling is required with fine steps of varying temperature gradient and bubble 
size in order to accurately delineate the parametric space and exact conditions for the onset of the various oscillatory modes for both space and ground-based applications.

\section{References}

1. Papazian, J. M. \& Wilcox, W. R., Interaction of Bubbles with Solidification Interfaces, AIAA J., Vol. 16, 447. 451, 1978.

2. Larkin, B.K., Thermocapillary Flow around Hemispherical Bubble, AIChE J., Vol. 16, No. 1, pp. 101-107, 1970.

3. Kao, Y.S. and Kenning, D.B.R., Thermocapillary Flow Near a Hemispherical Bubble on a Heated Wall, J. F. Mech., Vol 53, pp. 715-735, 1972.

4. Raake, D, Siekmann, J., Chun, Ch.-H., Temperature and Velocity Fields Due to Surface Tension Driven Flow, Exp. in Fiuids, Vol. 7, pp. 164-172, 1989.

5. Chun, C.-H., et al., Oscillating Convection Modes in the Surroundings of an Air Bubble Under a Horizontal Heated Wall, Experiments in Fluids, Vol. 11, 359-367, 1991.

6. Rashidnia, N., Observation of Flow and Temperature Oscillations Around a Bubble on a Solid Surface Subject to a Vertical Temperature Gradient, AIAA Paper, No. 95-0880, 1995.

7. Vest, C.M., Holographic Interferometry, Wiley, New York, 1979.

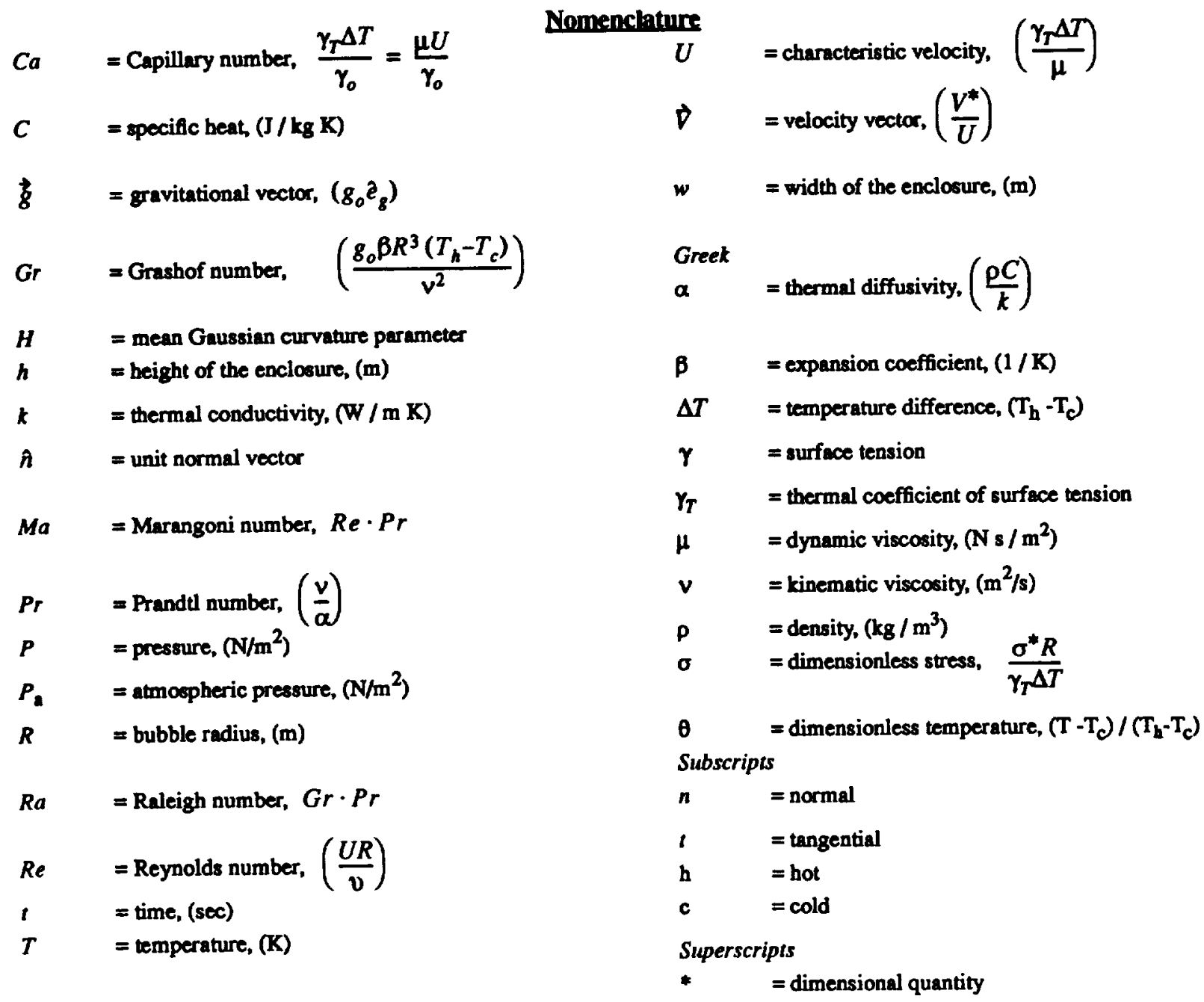




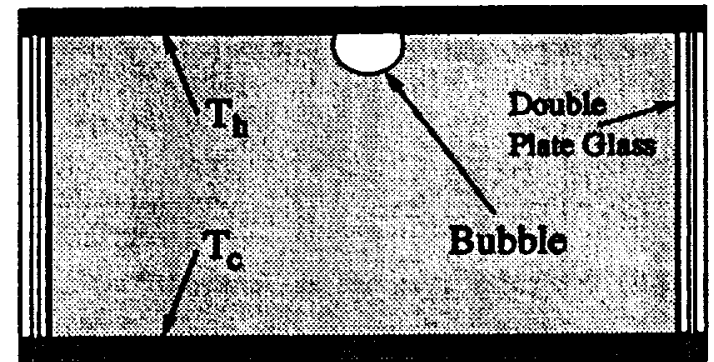

Figure 1. Schematic of The Test Enclosure.

a) Streamlines

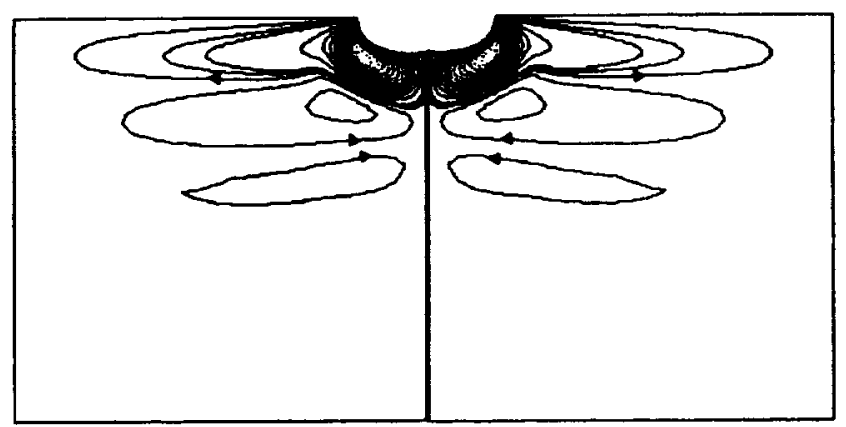

$\downarrow$

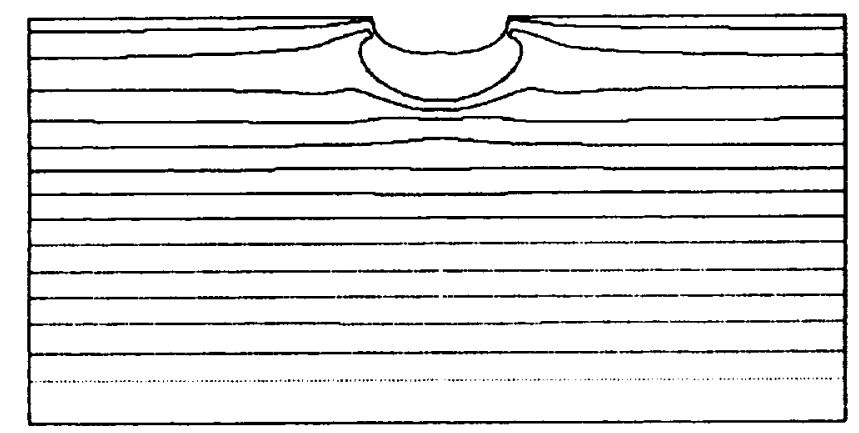

c) Bubble Shape

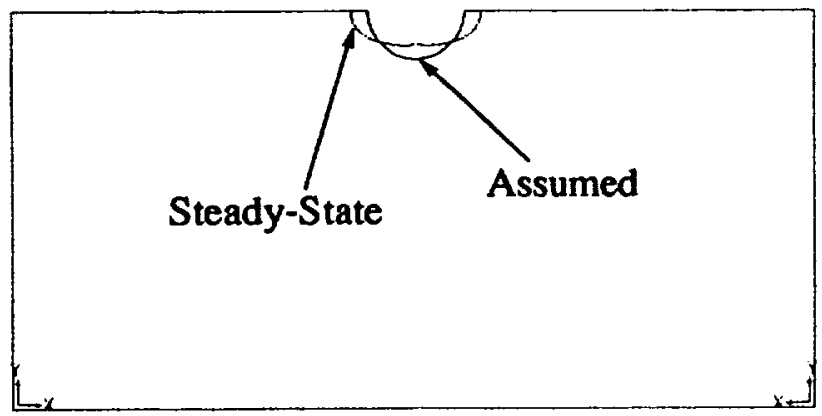

Figure 3. 1-G Numerical Predictions of Temperature and Flow Fields and Bubble Shape at Steady-State $(\mathrm{Re}=$ $4.3 \times 10^{3}, \mathrm{Gr}=2.4 \times 10^{3}$ ).

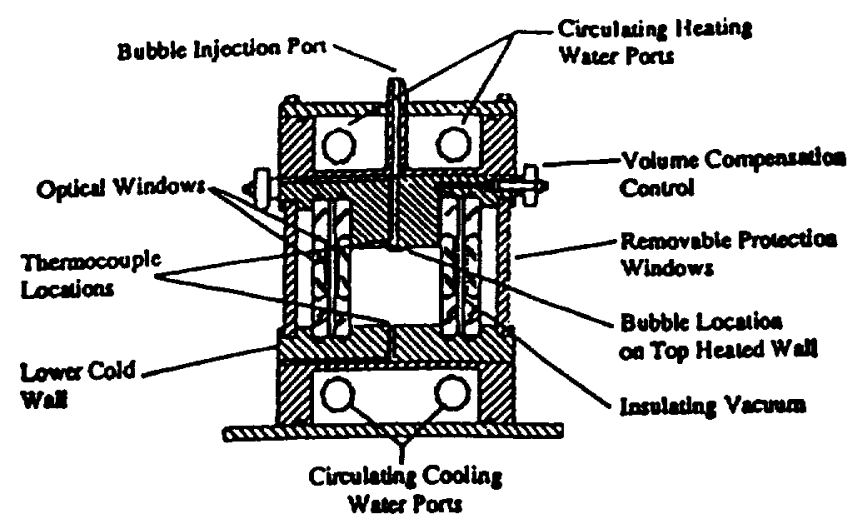

Figure 2. Schematic of The Test Cell

a) Streamlines

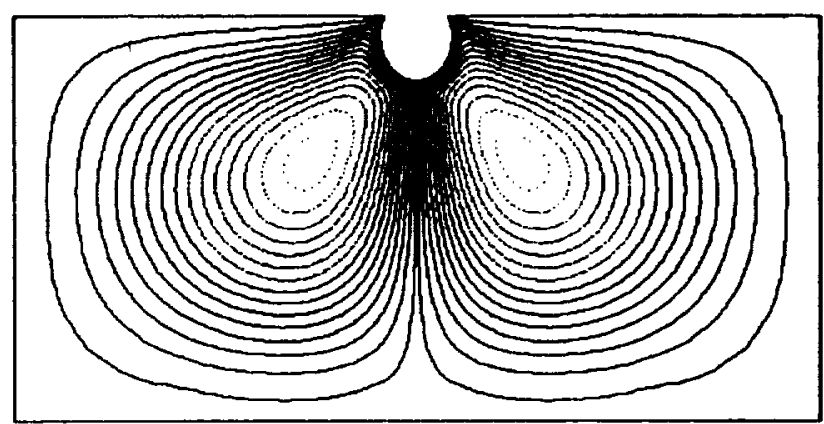

18

b) Temperature Contours

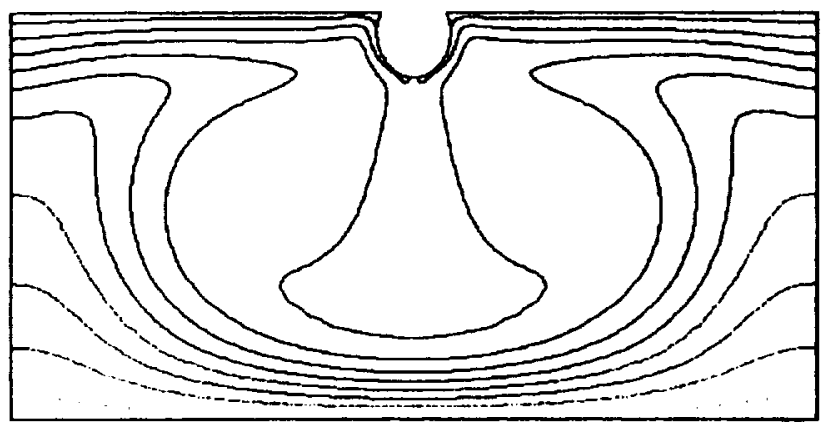

c) Bubble Shape

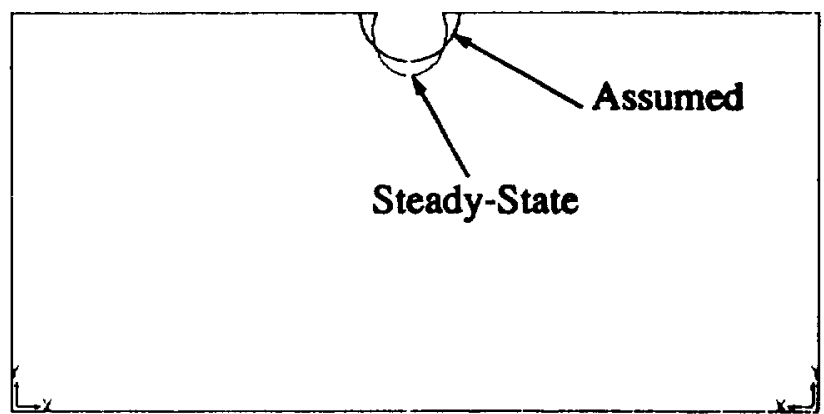

Figure 4. Low-G Numerical Predictions of Temperature and Flow Fields and Bubble Shape at Steady-State ( $R e=$ 430, $\operatorname{Pr}=8.4$ ). 


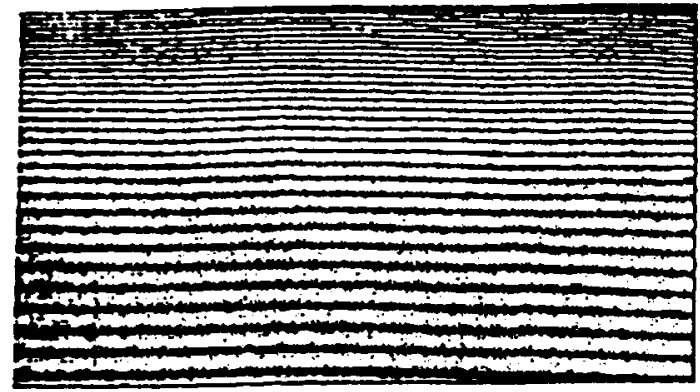

(a.

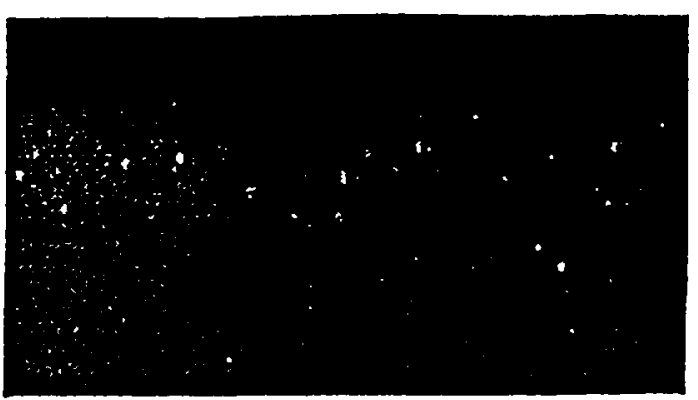

(b)

Figure 5. Steady-State 1-G Interferometric Fringe Patterns (a) and Laser Sheet Images (b) Depicting The Thermally Stratified Conditions Before Bubble Placement.

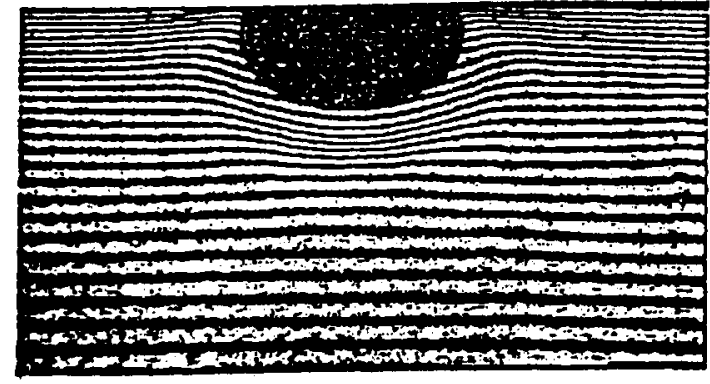

(a)

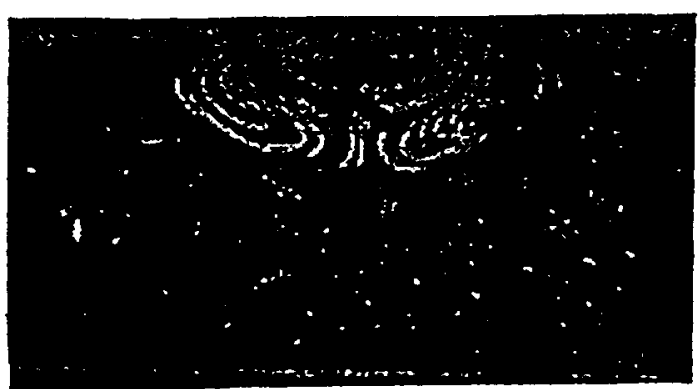

(b)

Figure 6. Steady-State 1-G Interferometric Fringe Patterns (a) and Laser Sheet Images (b) After Bubble Placement $\left(\mathrm{Ma}=3.6 \times 10^{4}\right)$. 

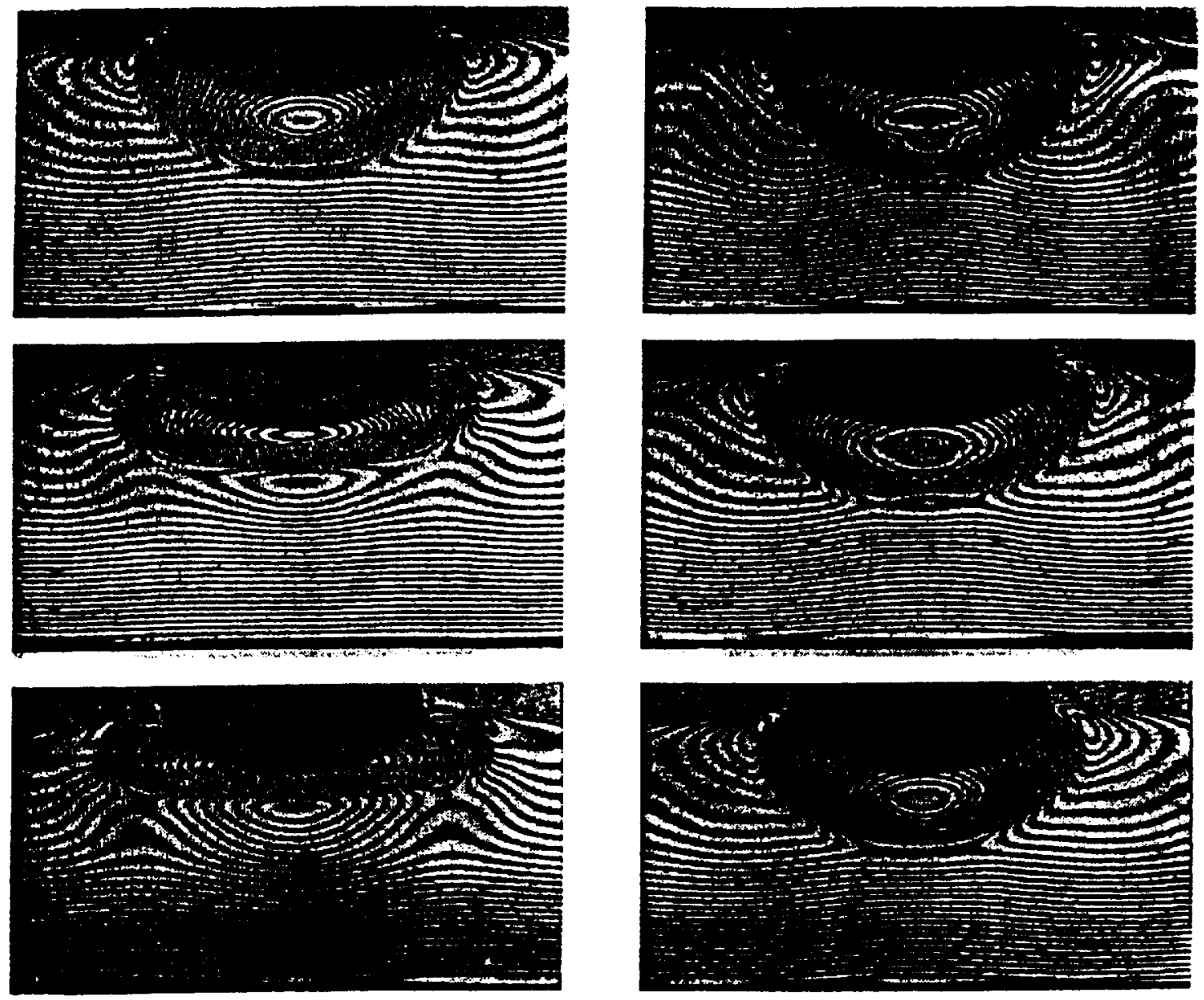

Figure 7. Sequence of Interferograms for Symmetric Oscillations of Temperature Field near the Bubble (Ma $\left.=9 \times 10^{4}\right)$. 